\section{Stanisław Iwańczak}

Rzym

stanwan2011@hotmail.it

DOI: http://dx.doi.org/10.12775/BPTh.2016.027

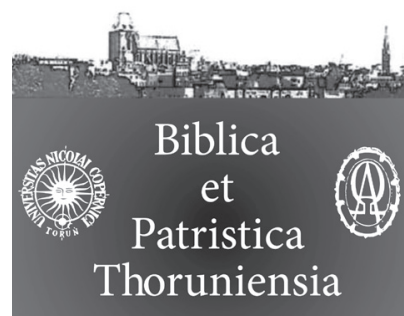

9 (2016) 3: 163-181

ISSN (print) 1689-5150

ISSN (online) 2450-7059

\title{
La controversia giuridica (rîb) di 2 Sam 11,27b-12,15a
}

\section{The juridical controversy (rîb) in 2 Samuel 11:27b-12:15a}

\section{Kontrowersja prawna (rîb) w 2 Sm 11,27b-12,15a}

Sommario. Lo studio di 2 Sam 11,27b-12,15a, fatto in questo articolo, presenta un bell'esempio del $r i ̂ b$ che è una controversia (contesa), la quale viene creata fra le due parti giuridiche e costituita dalle tre fasi (o tre elementi): accusa, risposta dell'accusato e riconciliazione (cfr. Tabella I). Nella fase iniziale-accusatoria della controversia si trova la triplice imputazione della contesa con la sua gradazione: Dio, Natan, Davide. La parte accusatoria dei versetti 11,27b-12,12 di 2 Sam è molto ricca e interessante dal punto di vista del rîb e del giudizio (cfr. Tabella II). Questo triplice processo accusatorio in 2 Sam $(A=11,27 b ; B=12,1 b-4 ; C=12,5 b)$ con la sua gradualità (Dio, profeta, re) e con la sua ripetizione $(A 1=12,7 b-12 ; B 1=12,7 a ; C 1=12,6 a)$ conduce subito alla confessione di Davide $(12,13 \mathrm{a})$, cioè nella risposta dell'accusato che è la seconda parte della controversia giuridica. Mentre i versetti 13b-14 sono la terza e ultima tappa del rîb in 2 Sam 12 che esprimono la riconciliazione tra Dio e Davide. Dio (accusatore) attraverso Natan (accusatore) concede il perdono a Davide (accusato e autoaccusatore).

Dall'analisi di 2 Sam 11,27b-12,15a risulta che Dio, nel rîb non si rivela come giudice severo, fra le due parti in conflitto, ma come accusatore, che non vuole vincere umiliando l'accusato, ma convincerlo e correggerlo. Egli si comporta come il membro, legato di una alleanza che desidera piuttosto salvare che condannare il suo alleato colpevole. Davanti all'accusatore misericordioso l'accusato ingiusto si apre e si dichiara reo, ammette la propria colpa e riceve alla fine il perdono. Il processo del rîb si conclude con la riconciliazione tra le due parti.

Riconoscere e confessare il proprio peccato, come ha fatto Davide (2 Sam 12,13a), è un grande atto di glorificazione del Signore, perché la confessione dei peccati diventa anche la professione di fede in Dio. Chi si confessa compie il più bel sacrificio di lode per Dio e nello stesso tempo manifesta il migliore atteggiamento davanti al Signore, perché la grandezza dell'uomo si esprime proprio nel dire la verità su se stesso. Il cuore contrito, aperto, sincero, umile durante la confessione non solo invoca il perdono di Dio, ma anche permette a Dio di salvare e liberare il peccatore dalle conseguenze del peccato e dalle sue sventure. Ecco il vero volto della misericordia divina che si basa 
sulla giustizia umana in conformità con la giustizia divina, e che si manifesta nella controversia giuridica, ossia nel rîb.

Abstract. The study of 2 Samuel 11:27b-12:15a, as conducted in the present article (paper), provides an eloquent example of the rîb, i.e. a controversy between two parties, which is made up of three stages (or elements): accusation, the reply of the accused, and reconciliation (cfr. Table I). In the initial stage of the dispute, i.e. the accusation, a threefold indictment is to be found, according to the following gradation: God, Nathan, David. The indictment part in verses 11:27b-12:12 is extremely rich and interesting when seen from the perspective of the rîb and the judgment (cfr. Table II). The threefold indictment process in 2 Samuel $(A=11: 27 \mathrm{~b} ; \mathrm{B}=12: 1 \mathrm{~b}-4 ; \mathrm{C}=12: 5 \mathrm{~b})$ with its gradation (God, prophet, king) and its repetition ( $\mathrm{A} 1=12: 7 \mathrm{~b}-12 ; \mathrm{B} 1=12: 7 \mathrm{a} ; \mathrm{C} 1=12: 6 \mathrm{a})$ leads directly to David's confession (12:13a), namely to the reply of the accused, which is the second stage of the legal dispute. Verses $13 \mathrm{~b}-14$ are the third and final stage of the rîb in 2 Samuel 12, and they express the reconciliation between God and David. God (accuser) through Nathan (accuser) grants his pardon to David (accused and selfaccuser).

The analysis of 2 Samuel 11:27b-12:15a shows that in the rîb God does not manifest himself as a severe judge between two litigants, but rather as an accuser who does not want to win humiliating the accused. In fact, He wants to convince and correct the accused. God behaves as the party to an alliance, who wishes to save rather than condemn his guilty ally. Before the merciful accuser, the party at fault opens up and declares himself guilty, admits his guilt and, in the end, is granted forgiveness. The rîb process ends with the reconciliation between the two parties.

Acknowledging and confessing one's sin, as David did (2 Samuel 12:13a), is an great act of glorification of the Lord, since the confession of sins also becomes a profession of faith in God. He who confesses performs the most beautiful sacrifice of praise to God, displaying at the same time the best attitude towards the Lord, given that man's greatness lies in telling the truth about oneself. A contrite, open, sincere and humble heart during confession not only invokes God's forgiveness but enables God to save and free the sinner from the consequences of sin and from his adversities. This is the true face of God's mercy, which rests on human justice as complying with divine justice, and which manifests in the juridical controversy, i.e. in the rîb.

Streszczenie. Studium 2 Sm 11,27b-12,15a, którego próba została podjęta w niniejszym artykule, przedstawia ciekawy przykład rîb, który jest sporem (kontrowersją) zaistniałym między dwoma stronami prawnymi i składającym się z trzech faz (lub trzech elementów): oskarżenia, zeznania oskarżonego oraz pojednania (por. Tabela I). W pierwszej fazie oskarżeniowej tej kontrowersji znajduje się potrójne oskarżenie razem ze swoją gradacją (stopniowaniem) ze strony Boga, Natana i Dawida. Część oskarżycielska znajdująca się w wersetach 2 Sm 11,27b-12,12 jest bardzo bogata i interesująca pod względem rîb oraz procesu prawnego (por. Tabela II). Ten potrójny proces oskarżycielski w $2 \mathrm{Sm}(\mathrm{A}=11,27 \mathrm{~b} ; \mathrm{B}=12,1 \mathrm{~b}-4$; $\mathrm{C}=12,5 \mathrm{~b})$ ze swoim stopniowaniem (ze 
strony Boga, proroka i króla) oraz ze swoim powtórzeniem (A1=12,7b-12; B1=12,7a; $\mathrm{C} 1=12,6 \mathrm{a})$ prowadzi do wyznania (przyznania się) Dawida $(12,13 \mathrm{a})$, to znaczy do zeznania oskarżonego, które jest drugą częścią (fazą) kontrowersji prawnej. Natomiast wiersze 13b-14 są trzecim i ostatnim etapem (fazą) rîb w 2 Sm 12, który wyraża pojednanie między Bogiem i Dawidem. Bóg (oskarżyciel) poprzez Natana (oskarżyciela) udziela przebaczenia Dawidowi (oskarżonemu i samooskarżycielowi).

Z analizy 2 Sm 11,27b-12,15a wynika, że Bóg w rîb nie objawia się (nie ukazuje się) jako surowy sędzia między dwoma stronami w konflikcie, ale jako oskarżyciel (oskarżający), który nie chce zwyciężyć czy też pokonać upokarzając oskarżonego, lecz przekonać go do poprawy i do zmiany jego postępowania. Bóg zachowuje się jako jedna ze stron przymierza, jako jego uczestnik, który pragnie raczej zbawić i uratować niż potępić czy skazać swojego winnego sprzymierzeńca (sojusznika). Przed oskarżycielem-miłosiernym oskarżony-niesprawiedliwy otwiera się i uznaje się za winnego, przyznaje się do własnego grzechu (winy, wykroczenia) i otrzymuje na koniec od niego przebaczenie. Proces rîb kończy się pojednaniem dwóch stron, oskarżyciela i oskarżonego.

Rozpoznać i wyznać własny grzech, jak to uczynił Dawid (2 Sm 12,13a), jest wielkim aktem uwielbienia Pana dlatego, że przyznanie się do grzechów i ich wyznanie staje się równocześnie wyznaniem wiary w Boga. Ten kto się spowiada składa najpiękniejszą ofiarę Bogu, a jednocześnie manifestuje najlepszy gest (postawę) przed Panem bowiem wielkość człowieka wyraża się właśnie wtedy kiedy wypowiada on prawdę o samym sobie. Serce skruszone, otwarte, szczere, pokorne podczas spowiedzi nie tylko prosi o przebaczenie Boga, ale także pozwala samemu Bogu wybawić i uwolnić grzesznika od konsekwencji grzechu oraz od jego nieszczęśliwych następstw. Oto prawdziwe oblicze Bożego miłosierdzia, które opiera się na sprawiedliwości ludzkiej zgodnej ze sprawiedliwością Bożą, a które ukazuje się w kontrowersji prawnej czyli w rîb.

Parole chiavi: controversia giuridica; rîb; contesa; accusa; autoaccusa; accusatore; accusato; giudice; giudizio; colpevole; colpa; peccato; confessione; perdono; riconciliazione; giustizia; parabola; Dio, re Davide; profeta Natan.

Keywords: juridical controversy; rîb; contestation; accusation; autoaccusation; accuser; accused; judge; judgment; guilty; guilt; sin; confession; forgiveness; reconciliation; justice; parable; David; prophet Natan.

Słowa kluczowe: kontrowersja prawna; rîb; spór; oskarżenie; samooskarżenie; oskarżyciel; oskarżony; sędzia; sąd; winny; wina; grzech; spowiedź-wyznanie; przebaczenie; sprawiedliwość; przypowieść; Bóg; król Dawid; prorok Natan. 
Tl tema di questo articolo è la presentazione di alcuni aspetti inerenti alla tematica del rîb in 2 Sam 11,27b-12,15a. Prima di entrare nel merito del lavoro, occorre una breve spiegazione del rî̀b. Uno dei suoi migliori approfondimenti sicuramente si trova nello studio Ristabilire la giustizia di P. Bovati nel quale l'autore lo definisce come segue: "Il rîb è una controversia che si viene a creare fra due parti su questioni di diritto; fra due persone, fra due gruppi o fra

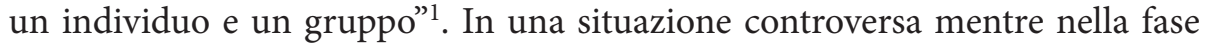
iniziale le due parti godono di una condizione pacifica, nel caso di un successivo episodio di abuso legale il rapporto normativo tra i due soggetti si rompe. Di conseguenza la parte lesa accusando l'altra parte di ingiustizia cerca una soluzione giuridica, conforme al diritto e rispettosa dell'accordo convenuto in precedenza. Il rîb si risolve quando le due parti ritrovano la concordia recipro$\mathrm{ca}$, secondo equità e giustizia, e ricostruiscono il loro rapporto iniziale di pace e d'alleanza ${ }^{2}$.

Sembra che il concetto del rîb con le articolazioni principali della sua controversia in modo globale e completo è stato presentato nello schema proposto dal sopracitato $\mathrm{P}$. Bovati ${ }^{3}$. In questo schema-definizione del rîb, presente qui sotto nella Tabella I, si trovano diverse varianti e varie modalità di soluzione della controversia.

Il primo passo nella controversia giuridica con il quale comincia il rîb è l'accusa della parte che si sente defraudata. In seguito questo processo si svolge tra l'accusato e l'accusatore. L'accusato ha due possibilità con quattro conclusioni: confessare la sua colpa o protestare la sua innocenza. Nel primo caso l'accusatore perdona la colpa dell'accusato e tutti due vanno insieme alla riconciliazione, oppure il primo non accetta la confessione dell'accusato e lo trascina davanti al giudice (tribunale). Nel secondo caso (seguono soluzioni simmetriche a quelle sopra, ma con motivazioni e sfumature diverse) l'accusatore non accetta l'innocenza dell'accusato e la causa finisce in tribunale, oppure quando l'accusatore è d'accordo sull'innocenza dell'accusato entrambi trovano il consenso reciproco senza ricorrere ad una terza istanza.

1 P. Bovati, Ristabilire la giustizia. pp. 21. Confronta in proposito anche B. Costacurta, Lo scettro e la spada. Davide diventa re (2 Sam 2-12), pp. 208-211 ("Il rîb o "controversia» è un procedimento giuridico di tipo bilaterale, in tutto si svolge solo tra la parte lesa e il colpevole, senza la mediazione decisoria del giudice").

2 Cfr. P. Bovati, I rîb profetici; P. Bovati, Ristabilire la giustizia, pp. 21-26; J. Vella, La giustizia forense di Dio.

3 Cfr. P. Bovati, Ristabilire la giustizia, pp. 23 (l'idea della Tabella I proposta da P. Bovati è stata qui leggermente modificata). 
L'ultima tappa della controversia tra i due - come si nota - può avere quattro conclusioni: due positive se l'accusatore si riconcilia con l'accusato, e due negative se il primo porta l'altro in tribunale.

\begin{tabular}{|c|c|c|c|}
\hline \multicolumn{4}{|c|}{ TABELLA I: CONTROVERSIA GIURIDICA } \\
\hline INIZIO & \multicolumn{2}{|c|}{ SVOLGIMENTO } & CONCLUSIONE \\
\hline Accusa & Accusato & Accusatore & Entrambi \\
\hline \multirow{4}{*}{$\begin{array}{l}\text { L’accusa } \\
\text { inizia il rîb }\end{array}$} & \multirow{2}{*}{$\begin{array}{c}\text { l'accusato confessa } \\
\text { la sua colpa }\end{array}$} & $\begin{array}{c}\text { l'accusa } \\
\text { concede } \\
\text { il perdono }\end{array}$ & RICONCILIAZIONE \\
\hline & & $\begin{array}{c}\text { l'accusa } \\
\text { rifiuta il perdono } \\
\text { (o le diverse forme } \\
\text { di transazione) }\end{array}$ & $\begin{array}{l}\text { TRIBUNALE } \\
\text { GUERRA }\end{array}$ \\
\hline & \multirow{2}{*}{$\begin{array}{l}\text { l'accusato protesta } \\
\text { la sua innocenza }\end{array}$} & $\begin{array}{l}\text { l'accusa } \\
\text { persiste } \\
\text { nell' } \\
\text { accusa }\end{array}$ & $\begin{array}{l}\text { TRIBUNALE } \\
\text { GUERRA }\end{array}$ \\
\hline & & $\begin{array}{c}\text { l'accusa } \\
\text { desiste } \\
\text { dall'accusa }\end{array}$ & RICONCILIAZIONE \\
\hline
\end{tabular}

Questa ricerca si baserà soprattutto sull'analisi filologica del testo 2 Sam $11,27 \mathrm{~b}-12,15 \mathrm{a}$ sotto l'aspetto della controversia giuridica senza esaminare il suo sfondo storico e la sua critica testuale ${ }^{4}$.

\section{Il contesto e la struttura di 2 Sam 11,27b-12,15a}

Nella lunga lista degli schemi anticotestamentari del rîb che propone G. Ravasi (Dt 32,1-25; Is 1,2-20; Mi 6,1-8; Ger 2,4-13.29; Is 42,18-25; 48,12-19; 57,3-13; 58,1-14; 66,1-4; Ger 6,16-21; Ml 1,6-2,9; Gdc 2,1-5; 1 Sam 2,27-36; 2 Sam $12,7-12 ; 1$ Re $14,7-11 ; 21,17-24)$ vi si vede anche la controversia giuridi-

4 Per approfondire le varie questioni letterarie e storiche del testo 2 Sam 12 può essere utile lo studio biblico di W. Oswald, Nathan der Prophet: Eine Untersuchung zu 2 Samuel 7 und 12 und 1 Könige 1, come pure A.G. Auld, I \& II Samuel; A.G. Auld - E. Eynikel, For and against David: Story and History in the Books of Samuel; D. Firth, 1 and 2 Samuel: A Kingdom Comes; R.P. Gordon, 1 \& 2 Samuel. 
ca sul doppio peccato di Davide nel secondo libro di Samuele ${ }^{5}$. Lo stesso 2 Sam 12 , come testo classico che riunisce i tre atti penitenziali (accusa, confessione, perdono) nel giudizio bilaterale, è citato pure da A. Schökel, in una serie di alcuni testi narrativi che ha analizzato (Nm 12,6-14; 21,4-9; 22,32-35; 1 Sam 15,14-26; 2 Sam 12,1-14; 1 Re 8,46-51; 2 Sam 24,1-12) dopo i Salmi 50 e 51, i quali formano anchessi una liturgia penitenziale ${ }^{6}$.

La narrazione dei capitoli 11-12 del secondo libro di Samuele con la sua interessante causa del rîb si compone generalmente di due parti: l'episodio Davide - Betsabea $^{7}$ (2 Sam 11,1-27) e le parole di Natan rivolte a Davide con gli eventi conseguenti (2 Sam 12,1-25). La prima parte è segnata dal doppio peccato di Davide, mentre la seconda lo rivela con le sue conseguenze. In questo "dittico" si può vedere da una parte la caduta di Davide e dall'altra il suo pentimento; gli atti peccaminosi ed il loro rimprovero; gli eventi cattivi ed il loro commento; quasi una tesi ed antitesi ${ }^{8}$. Come suggerisce Jones ${ }^{9}$ il versetto 27 a è un climax del capitolo 11, invece il 27b è un anello redazionale tra i capitoli 11 e 12 . Il capitolo 12 si divide pure in due: la cosiddetta sezione profetica ([11,27b]12,1$-14[15 \mathrm{a}])$ e la sua conseguenza $(12,15[15 \mathrm{~b}]-25)^{10}$. Giustamente alcuni ${ }^{11}$ notano che il versetto $27 \mathrm{~b}$ del capitolo 11 non solo unisce l'altro capitolo, ma anche inizia la sezione profetica, invece il v. 15 a del cap. 12 tematicamente conclude questa sezione: "Natan tornò a casa" (wayyēlek nātān 'el-bêtôô) ${ }^{12}$.

Dal brano di 2 Sam 11,27b-12,15a si possono staccare abbastanza nettamente sia i tre momenti della controversia giuridica proposta da Bovati ${ }^{13}$ che i tre atti della liturgia penitenziale suggerita da Schökel ${ }^{14}$ : inizio-accusa $(11,27 b-$ -12,12); svolgimento-confessione (12,13a); conclusione-perdono (12,13b-15a).

5 Cfr. G. Ravasi, Il Libro dei Salmi (I), pp. 902.

6 Cfr. A. Schökel, Trenta Salmi, pp. 239-244 (sia Sal 50 che Sal 51 costituiscono due parti, accusatore e accusato, di una liturgia penitenziale composta da una requisitoria divina, confessione e perdono).

7 Lo studio biblico approfondito sulla persona di Betsabea si trova nel libro di S.M. Koenig, Isn't this Bathsheba? A study in characterization.

8 Cfr. A.A. Anderson, 2 Samuel, pp. 144; G.H. Jones, The Nathan narratives, pp. 95; P.K. McCarter, II Samuel, pp. 304-309.

9 Cfr. G.H. Jones, The Nathan narratives, pp. 96.

10 Cfr. A.A. Anderson, 2 Samuel, pp. 161.

11 Cfr. ibidem, pp. 160-161; G.H. Jones, The Nathan narratives, pp. 108.

12 La trascrizione di tutte le parole ebraiche in questo lavoro si fa secondo T. O. Lambdin, Introduction to Biblical Hebrew. Cfr. anche P.P. Joüon, Grammaire de l'hébreu biblique.

13 Cfr. P. Bovati, Ristabilire la giustizia, pp. 23.

14 Cfr. A. Schökel, Trenta Salmi, pp. 210-211 ("Un giudizio bilaterale si sviluppa tra le due parti in una contesa, senza appello ad un giudice o ad un terzo, benché, a volte, davanti 


\section{L'inizio della controversia giuridica (2 Sam 11,27b-12,12)}

Lanalisi si sofferma adesso sulla prima parte, presentata sopra, relativa alla divisione che è più lunga e più allargata. In essa si trova la triplice imputazione della contesa con la sua gradazione: Dio, profeta, re.

\subsection{Il discorso accusatorio di Dio ( 2 Sam $11,27 b ; 12,7 b-12)$}

Dopo la vera storia di Davide (2 Sam 11,2-27) che commette il doppio peccato verso Uria (lo uccide in modo perverso tramite il suo ordine: "Ponete Uria in prima fila, dove più ferve la mischia; poi ritiratevi da lui perché resti colpito e muoia" - 2 Sam 11,15.17) e verso sua moglie Betsabea (l'adulterio: "Allora Davide mandò messaggeri a prenderla. Essa andò da lui ed egli giacque con lei..." - 2 Sam 11,4a), il narratore alla fine del racconto aggiunge in 2 Sam 11,27b: "Ma ciò che Davide aveva fatto era male agli occhi del Signore" (wayyèra' haddābār

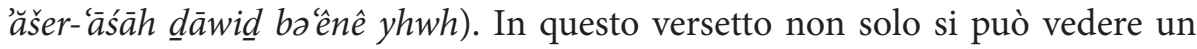
ponte ${ }^{15}$ verso il capitolo successivo e un remesie di tutto il capitolo 11, ma anche si può osservare che le sue parole diventano unaccusa ${ }^{16}$, una imputazione da parte di Dio nei confronti di Davide che aveva agito male davanti al Signore.

La frase simile, con piccole variazioni, quasi si ripete anche in 2 Sam 12,9a: "Perché17 dunque hai disprezzato la parola del Signore, facendo ciò che è male ai suoi occhi?" (laăśốt hära bə 'ênaw). La seconda parte dello stesso versetto (2 Sam 12,9b) precisa il reato di Davide cioè il suo doppio peccato che non

a testimoni notarili"). Nel brano analizzato si può osservare anche, in certo modo, un giudizio bilaterale tra Dio che si sente defraudato da parte del suo re (2 Sam 12,7b-8) e Davide che ha offeso il suo Signore attraverso il doppio peccato (2 Sam 12,9). La voce di Dio, la sua accusa rivolta a Davide, si prolunga nella persona di Natan, che è il profeta, il portavoce di Dio. Lazione profetico-giuridica di Natan in 2 Sam 12,1-15a, le sue parole appartengono a Dio. Lui diventa un mezzo, lo strumento del Signore. Dall'altra parte si può vedere in Uria (povero della parabola) e Betsabea la parte pure defraudata, invece in Dio (Natan) la terza istanza giuridica che li difende.

15 Cfr. G.H. Jones, The Nathan narratives, pp. 96.

16 Cfr. P. Bovati, Ristabilire la giustizia, pp. 51 ("L'accusa può formalizzarsi quando un soggetto giuridico [nel caso analizzato sopra - Dio] imputa ad una determinata persona [Davide] la responsabilità di un atto [il doppio peccato di Davide] non conforme al diritto o vietato dalla legge").

17 Cfr. ibidem, pp. 63-64 (Ci sono due forme in cui si esprime la parola di accusa: in forma dichiarativa oppure sotto forma interrogativa. Il versetto 9a appartiene alla seconda forma, poiché comincia dalla domanda introdotta dall'avverbio interrogativo maddūa [ cfr. pure Es 1,18; Lev 10,27; 1 Re 1,6]). 
piace al Signore: "Tu hai colpito di spada Uria l'Hittita, hai preso in moglie la moglie sua e lo hai ucciso con la spada degli Ammoniti" (cfr. 2 Sam 11,4a.1517). Analizzando il contesto di questi due delitti (2 Sam 12,9b-10) e delle due espressioni accusatorie pronunciate dal Signore (2 Sam 12,9a.10b) sembra che questo doppio reato era un frutto del disprezzo (rifiuto) del Signore da parte di Davide. Quindi si può dedurre che inizialmente la mancanza del suo rispetto per il Signore ha causato in seguito il suo doppio peccato ${ }^{18}$. Per questo disdegno il Signore lo accusa utilizzando le espressioni di imputazione, prima in 2 Sam 12,9a: "Perché dunque hai disprezzato la parola del Signore" (maddūa' bāzît $\bar{a}$ 'et-dabar $y h w h$ ), e poi in 2 Sam 12,10b: "poiché tu mi hai disprezzato" ('éqeb kî bazițānî). Da una parte si può notare che il versetto $10 \mathrm{~b}$ (accanto al peccato del disprezzo appena analizzato) ricorda e ripete solo il peccato di adulterio di Davide con Betsabea, ma dall'altra parte sembra che il versetto $12 \mathrm{a}$ indichi e riveli il suo altro peccato che è perverso e nascosto, cioè l'assassinio di Uria ("poiché tu l'hai fatto in segreto") ${ }^{19}$.

Bisogna ancora accennare che nel brano analizzato (2 Sam 11,27b-12,12) si trovano le due forme (o due modi) del comportamento giuridico di Dio. Se questo comportamento in 2 Sam 11,27b è stato descritto per mezzo del narratore, in 2 Sam 12,9-10.12 esso viene direttamente messo sulla bocca del Signore come indica la frase: "Così dice il Signore" (kōh 'āmar yhwh), la quale in 2 Sam $12,7 \mathrm{~b}$ e in 2 Sam 12,11 introduce i due oracoli ${ }^{20}$. Quindi sia il versetto $11,27 \mathrm{~b}$ che i versetti 12,7b-12 di 2 Sam si possono nominare insieme come parte accusatoria di Dio (come suo discorso di imputazione) rivolto a Davide.

18 Una simile osservazione presenta nel suo articolo D. Janzen, The condemnation of David's "taking" in 2 Samuel 12:1-14, pp. 209-220. Il disprezzo di Dio - secondo D. Janzen diventa la motivazione per il doppio crimine di Davide. Infatti quando Davide si confessa in 2 Sam 12,13a dice "Ho peccato contro il Signore", non contro Uria e Betsabea. Questa ribellione contro Dio viene confermata anche dalle parole di Natan: "tu hai insultato il Signore" (2 Sam 12,14).

19 La seconda parte del versetto 12 è molto interessante dal punto di vista giuridico ("ma io farò questo davanti a tutto Israele e alla luce del sole"). Da un lato Dio "convoca" per i suoi testimoni la terra ("tutto Israele") e il cielo ("la luce del sole"), ma dall'altro lato rivela alla luce del sole (la luce diviene simbolo della vittoria della giustizia) il peccato nascosto di Davide davanti a tutto Israele (cfr. P. Bovati, Ristabilire la giustizia, pp. 203-204, 339; B. Costacurta, Lo scettro e la spada, pp. 202-203).

20 Cfr. G.H. Jones, The Nathan narratives, pp. 101-106. 


\subsection{Gli interventi profetico-accusatori di Natan ( 2 Sam $12,1 b-4 ; 12,7 a)$}

All'interno della cornice sopra descritta se ne trova ancora un'altra interna (12,1b-4 e 12,7a del 2 Sam), la quale rispecchia i due interventi profetici di Natan che cominciano con simili espressioni. Nel versetto 12,1b: "[Natan] andò da lui e gli disse" (wayyābōō' [nātān] 'élâw wayyō'mer lô $)^{21}$, invece nel versetto 12,7a: "Allora Natan disse a Davide" (wayyō'mer nātān 'el-dāwid). In entrambe le frasi il verbo caratteristico, come si vede, è "disse" (wayȳ' 'mer) ${ }^{22}$.

La prima accusa di Natan contro Davide, indiretta e provocatoria, viene espressa nella forma fittizia ${ }^{23}$ di una parabola. Questa parabola ${ }^{24}$ come un caso fittizio (come un artificio) in poche parole descrive velatamente l'abuso per-

21 Il versetto $12,1(1 \mathrm{a}+1 \mathrm{~b})$ è molto interessante sotto l'aspetto giuridico. In esso da una parte si vede l'azione divina nella quale Dio - il primo accusatore di Davide $(11,27 \mathrm{~b})$ mandò (wayyišlah) il suo portavoce, il profeta Natan, a Davide - il colpevole, ma dall'altra parte si osserva in questo versetto l'azione profetica dello stesso Natan - il difensore (avvocato) del povero che andò (wayyāb $\left.\underline{b} \bar{o}^{\prime}\right)$ al re, alla suprema istanza giudicante, per presentare la causa. Nell'azione di Natan si esprime il "movimento" verso il tribunale perché - come spiega Bovati - "l'iniziativa delle parti in causa è espressa generalmente mediante dei verbi di movimento: «soggetto grammaticale» del verbo è colui (Natan) che sottopone il caso o denuncia il colpevole; il «terminus ad quem» è la persona o funzione giudicante a cui (Davide) ci si rivolge. La struttura giuridica comporta sempre tre elementi: le due parti (ricco-Davide; povero-Uria) e il giudice (Davide)" (P. Bovati, Ristabilire la giustizia, pp. 198). Nel caso qui analizzato chi assume l'iniziativa è Natan (però non si può dimenticare che Dio è sempre il primo iniziatore di tutta questa azione) perché si muove, si rivolge (wayyābōó') verso l'istanza giudicante che è il re Davide ('êlâw = "da lui" cioè "da Davide" = 'el-dâwid). Cfr. B. Costacurta, Lo scettro e la spada, pp. 195-196.

22 Questo verbo che è al futuro Qal 3 persona singolare maschile, proviene dalla radice ' $m r$ ed esprime l'idea del "dire, parlare"; spesso viene usato per introdurre un discorso.

23 Nella controversia a due è interessante l'uso, o meglio la presentazione di un caso fittizio nel quale l'accusato è chiamato a pronunciare un verdetto. Mediante questo artificio, senza ricorrere ad un terzo soggetto giuridico, l'accusa giunge a mostrare la verità delle sue affermazioni. In questo caso il desiderio dell'accusa non è di vincere l'accusato, ma piuttosto di convincerlo. Cfr. P. Bovati, Ristabilire la giustizia, pp. 69.

24 Cfr. U. Simon, The poor man's ewe-lamb, pp. 207-242. La parabola sulla pecorella del povero del 2 Sam 12,1b-4 rappresenta un esempio tipico di ben definita categoria delle parabole bibliche che possono essere chiamate parabole giuridiche. Secondo lo stesso biblista ci sono cinque parabole di questo genere letterario nella Bibbia: tre nei libri storici (2 Sam 12,1-7; 14,1-20; 1 Re 20,35-43) e due nei libri profetici (Is 5,1-7; Ger 3,1-5). La parabola giuridica è la parabola travestita che soccorre l'avvicinarsi dell'umo a se stesso e che permette a lui di fare un autogiudizio con lo stesso criterio che lui applica agli altri. Le preziose osservazioni sulla stessa parabola propone anche B. Costacurta, Lo scettro e la spada, pp. 195-200, nonché D. Bodi, The demise of the warlord, pp. 71-78, interpretandola alla luce di alcuni testi accadici dell'antico Vicino Oriente. 
verso del re (2 Sam 12,1b-4). La seconda imputazione del profeta, più breve, è rivolta direttamente a Davide (2 Sam 12,7a), diventa contemporaneamente sia accusa centrale contro il re di tutta la parte accusatoria (2 Sam 11,27b-12,12) ${ }^{25}$ sia motivo principale per la confessione del sovrano nel versetto 13 a di 2 Sam 12. Le parole di Natan destinate a Davide attraverso la parabola raggiungono il loro climax nella semplice applicazione del versetto 7a di 2 Sam 12: "Tu sei quell'uomo!" ('attāhh hā' 'î́s) ${ }^{26}$. Quando il ricco "prese la pecora" (2 Sam 12,4) senza pagare, il povero avrebbe potuto rivolgere l'accusa contro colui (quel primo) che si era approfittato della sua posizione, per mostrare la trasgressione della legge; ma il caso doveva essere presentato dalla terza persona, il profeta. Questo era un caso che contravveniva la legge e in quanto tale era portato da Natan al re che era l'istanza suprema della legge ${ }^{27}$. In tale situazione (processo, caso) il re non avrebbe potuto non difendere il povero, dunque pronunciare giustamente il suo verdetto (2 Sam $12,5-6)^{28}$.

\subsection{Davide e le sue autoaccuse ( 2 Sam $12,5 b ; 12,6 b$ )}

Proprio al centro del quadro accusatorio-processuale sopra disegnato (2 Sam 11,27b-12,12) si trova la reazione (2 Sam 12,5a) e quel verdetto di Davide (2 Sam 12,5b.6) che, come re, è il supremo tribunale monarchico, la suprema giurisdizione su tutto Israele, il tribunale reale ${ }^{29}$. Da una parte il re Davide dà la sua sentenza ${ }^{30}$ sull'abuso vizioso del ricco, che nello stesso tempo è l'autocon-

25 Cfr. P.K. McCarter, II Samuel, pp. 307 ("Il climax della storia nei cap. 10-12 viene in 12,7a [«Tu sei quell'uomo!»] quando le implicazioni degli atti di Davide sono state rivelate”). Le parole di Natan: 'attēh hā' 'îs esprimono pure in certo modo un giudizio, una sentenza. Da una parte queste parole sono "accusa" perché "tu sei quell'uomo" che ha ucciso Uria e ha commesso adulterio con Betsabea, ma dall'altra parte esse sono anche "giudizio, sentenza" perché "tu sei quell'uomo" che è il colpevole di quelli delitti (cfr. P. Bovati, Ristabilire la giustizia, pp. 328-329; vedi anche la nota 16 di questo articolo che presenta la definizione di accusa e il paragrafo 4 con le note 56-63 che mostrano la sentenza).

26 Cfr. G.H. Jones, The Nathan narratives, pp. 96.

27 Cfr. A. Schökel, Trenta Salmi, pp. 213 (“Oltre il re.., in Israele non c'era un'istanza giudiziaria superiore alla quale si poteva ricorrere, perché il re era il giudice supremo").

28 Cfr. G.H. Jones, The Nathan narratives, pp. 100.

29 Cfr. P. Bovati, Ristabilire la giustizia, pp. 25; B. Costacurta, Lo scettro e la spada, pp. 199 ("E se anche percepisce la dimensione simbolica del racconto profetico, Davide si sente messo in gioco nel suo ruolo di sovrano, garante della giustizia e giudice giusto").

30 In realtà Davide ha pronunciato le due sentenze. Secondo P. Bovati la prima (v. 5b) appartiene al tipo "è reo di ... (morte)", invece la seconda (v. 6) si trova nel gruppo "condannato a morte" (cfr. P. Bovati, Ristabilire la giustizia, pp. 329-334; di più sulle sentenze vedi anche il paragrafo 4 con le note 56-63 di questo articolo). Da un lato queste sentenze di 
danna per la sua ingiustizia verso Uria e Betsabea, ma dall'altra parte, il suo giudizio diventa una conferma delle accuse precedenti e seguenti di Dio $(11,27 \mathrm{~b}$ e 12,7b-12 di 2 Sam) e di Natan (12,1b-4 e 12,7a di 2 Sam) contro di lui. Questo suo autogiudizio diventa anche, in un certo senso, "la sua autoaccusa" (2 Sam $12,5 \mathrm{~b}$ e $6 \mathrm{a})^{31}$.

La descrizione nel versetto 3 (2 Sam 12) dell'affetto del povero verso la sua pecorella era voluta per svegliare le emozioni dell'ascoltatore; il suo successo è dimostrato nell'impulsività della rabbia di Davide al versetto 5a (2 Sam 12), e dunque nel suo verdetto (2 Sam 12,5b). Natan ha descritto la situazione con tanta vivacità che Davide era quasi costretto a pronunciare un giudizio ${ }^{32}$ sul ricco. Il suo giudizio non è dichiarato chiaramente, perché inizialmente nel 2 Sam 12,5b il re ha pronunciato la sentenza di morte: "Chi ha fatto questo merita la morte" (kî ben-māwet hā'î̌s hāóóseh $z \bar{o} \underline{t})^{33}$, la quale in 2 Sam 12,6a (cf. Es

Davide sembrano finali, perché sono pronunciate dal re (il supremo tribunale), ma dall'altro lato sono parziali, perché dal contesto risulta che Dio è l'ultimo che annuncia la sua sentenza (v. 13-14).

31 Per approfondire meglio le parole di Davide servirà in questo punto del lavoro una precisazione dei due termini "accusa" e "sentenza", e della loro reciproca relazione, tanto più che in questa prima sezione del rîb (2 Sam 11,27b-12,12) attualmente analizzata, tutti e tre i pronunciamenti (di Dio, di Natan, di Davide) sono trattati come le accuse. Spiegando (precisando) il rapporto dellaccusa con la sanzione e il loro significato secondo P. Bovati: "possiamo così affermare che una accusa risponde veramente alla sua natura se, non solo si limita a contestare il reato, ma si avvale specificamente del potere concreto di applicare al colpevole una adeguata punizione. È evidente che da un punto di vista logico, accusa e sanzione sono distinte $[\ldots]$. Ciò nonostante, si può affermare che essere sotto accusa equivale di fatto ad essere sotto la minaccia di una qualche sanzione, così come «accusare» $\mathrm{O}$ «intraprendere una azione punitiva» nei suoi confronti [...]. Latto dell'accusa si presenta spesso come fosse una sentenza (un verdetto giudiziario) nei confronti dell'imputato; [...] laccusa spessissimo non riceve risposta, e talvolta è immediatamente articolata allatto concreto della punizione, quasi ci trovassimo in presenza di un giudice che consegna l'accusato, riconosciuto colpevole, nelle mani dell'esecutore di giustizia. La somiglianza nel tenore espressivo tra queste due formalità del procedere giuridico non deve tuttavia far dimenticare la differenza radicale tra di esse, differenza che così enunciamo: laccusa è sempre l'anticipazione condizionale della sentenza" (P. Bovati, Ristabilire la giustizia, pp. 70-77). Prendendo in considerazione sia la spiegazione di Bovati sia la posizione delle sentenze di Davide nel loro contesto biblico (specialmente il loro posto e anticipazione in relazione alla sentenza finale di Dio nei versetti 13-14) in certo senso si può trattare le parole di Davide come accuse (v. 5a-6).

32 Il sinonimo del termine "giudizio", tra l'altro, è "sentenza, verdetto" e viceversa sotto la voce "sentenza" si trova "giudizio e verdetto" (cfr. S. Gioda, Dizionario, sinonimi e contrari, pp. 185, 376).

33 Cfr. P. Bovati, Ristabilire la giustizia, pp. 330. 
21,37) era ammorbidita ed espressa nel pagamento monetario: "Pagherà quattro volte il valore della pecora" (wə'et-hakkibəśāh yəšallēm 'araba'attāyim) ${ }^{34}$. Natan presentando la parabola-accusa ${ }^{35}$ aspettava da Davide il suo verdetto sul ricco. Un semplice caso di furto non richiedeva una sentenza di re (reale), perché esso poteva essere risolto nel cercare la giustizia alla porta ${ }^{36}$. La pena per tale reato (delitto) era chiaramente definita dalla legge ${ }^{37}$, invece l'indignazione mostrata da Davide nella sua prima risposta (2 Sam 12,5b) può suggerire che questo caso era più grave di quello che presenta la lettura superficiale del testo della parabola ${ }^{38}$. Queste difficoltà possono far capire che lo scopo della parabola era semplicemente "cavare fuori" da Davide le parole della condanna"; ${ }^{39}$ con tali parole, egli ha partecipato al processo di autocondanna ${ }^{40}$. Lo scopo è stato

34 Cfr. ibidem, pp. 334; B. Costacurta, Lo scettro e la spada, pp. 199 ("Nel motivare la propria sentenza, Davide afferma che l'uomo da condannare «non ha avuto pietà» ( 1 Samuel 16,1-1 Rois 2,11, pp. 232.

35 Questa parabola è diventata una accusa indiretta (vedi la nota 20).

36 Cfr. G.H. Jones, The Nathan narratives, pp. 99.

37 Cfr. H. Simon, The poor man's ewe-lamb, pp. 227-228. H. Simon, l'autore della Pecora-agnello del povero uomo per spiegare meglio il concetto della parabola sulla pecora prova a trovare qualche esempio dei costumi o delle tradizioni che assomigliano alla scena del 2 Sam 12,1-4. Analizzando diversi testi lui si ferma sull'opera di Aref el Aref dove c'è l'Adayeh cioè una forma beduina di furto legale. Tra le varie regole dell'Adayeh che permettono o proibiscono ce ne sono alcune che ci interessano di più. Nel caso di un ospite inatteso era permesso prendere una pecora dal gregge di un vicino, avvisando il proprietario. Alcuni animali erano esenti da quella legge, tra loro era esentata una pecorella allevata in una tenda per la quale il proprietario aveva un affetto speciale. Se qualcuno aveva preso questo tipo di animale doveva pagare quattro volte. Può essere probabile che Natan nell'incontro con Davide abbia utilizzato questa legge nella sua parabola.

38 Una simile spiegazione del primo verdetto è proposta pure da A.A. Anderson. Secondo lui la falsa ospitalità del ricco che sfrutta il povero, la condizione perversa del ricco, la sua perversità è praticamente degna di morte; tale uomo sembra essere non solo un ladro, ma anche un adultero e un assassino (cfr. A.A. Anderson, 2 Samuel, pp. 160). Invece J. Schipper analizzando queste due risposte di Davide (2 Sam 12,5-6) argomenta nel suo articolo che piuttosto Davide non ha interpretato la parabola di Natan come intendeva il profeta (cfr. J. Schipper, Did David overinterpret Nathan's parable in 2 Samuel 12:1-6?, pp. 383-407).

39 Il sinonimo della "condanna", è fra gli altri, "sentenza" (cfr. S. Gioda, Dizionario, sinonimi e contrari, pp. 109).

40 Cfr. B. Costacurta, Lo scettro e la spada, pp. 200 ("il racconto profetico raggiunge la coscienza del peccatore che ... reagisce secondo verità e, condannando il male altrui, si autocondanna”). 
raggiunto: l'ascoltatore - il supremo giudice, che non sospetta niente, passa all'autogiudizio ${ }^{41}$.

Il punto centrale di questo incontro tra Natan e Davide è l'annuncio del verdetto reale che provenendo da lui stesso diventa il suo autoverdetto. Lapplicazione improvvisa di Natan: “Tu sei quell'uomo!" (2 Sam 12,7a) crea un parallelismo ${ }^{42}$ tra lo sfruttamento del povero da parte del ricco (2 Sam 12,1-4) e il cattivo uso della posizione (dell'autorità) del re (2 Sam 11,2-27). Quindi Davide viene a trovarsi in una situazione molto problematica, perché da una parte è come l'oppressore ricco (2 Sam 12,1-4), ma dall'altra parte diventa come il giudice supremo (2 Sam 12,5-6). Come re lui amministra la giustizia; come ricco oppressore sovverte quella giustizia. Davide, il giudice supremo, condanna Davide, il ricco oppressore. Il problema giuridico del capitolo 11 di 2 Sam si risolve, il giudizio è tirato fuori, il processo è quasi finito ${ }^{43}$.

Se si prende in considerazione che Natan è profeta ${ }^{44}$ e messaggero di Dio, perché è stato mandato dal Signore (2 Sam 12,1a), e che Davide è il re, prima scelto (2 Sam 7,8), poi unto dal Signore (2 Sam 12,7b) e supremo giudice ${ }^{45}$ in Israele (2 Sam 12,5-6; cfr. 1 Re 3,9.11b), si può notare che in entrambi, ci sono le istanze supreme e immediate di Dio riguardo al nuovo regno israelitico. Quindi nel profetismo di Natan e nella regalità di Davide si può vedere l'azione giuridico-accusatoria indiretta di Dio su Davide che si realizza e si personalizza nel profeta e nel re.

Dopo l'analisi esegetica del triplice giudizio-accusa con la sua gradazione (perché da parte del Signore, del profeta e del re) e con la sua ripetizione quasi simmetrica nel testo di 2 Sam 11,27b-12,12 si può esprimere un seguente schema processuale (accusatorio): Dio $=\mathrm{A}(11,27 \mathrm{~b}), \mathrm{A}^{1}(12,7 \mathrm{~b}-12)$; Natan $=$ $\mathrm{B}(12,1 \mathrm{~b}-4), \mathrm{B}^{1}(12,7 \mathrm{a})$; Davide $=\mathrm{C}(12,5 \mathrm{~b}), \mathrm{C}^{1}(12,6)$. Questo schema è stato presentato, qui sotto, nella Tabella $\mathrm{II}^{46}$.

41 Cfr. H. Simon, The poor man's ewe-lamb, pp. 220 (sulla funzione della parabola giuridica).

42 Cfr. G.H. Jones, The Nathan narratives, pp. 100.

43 Cfr. P.K. McCarter, II Samuel, pp. 298-299.

44 Cfr. J.L. Sicre, I profeti d'Israele e il loro messaggio, pp. 44-48 (tra alcune caratteristiche della personalità profetica, secondo lui, un profeta deve essere un uomo ispirato, un portavoce del Signore).

45 Cfr. P. Bovati, Ristabilire la giustizia, pp. 25.

46 Cfr. G. Ravasi, Il Libro dei Salmi (II), pp. 26-27. 
TABELLA II: SCHEMA PROCESUALE (ACCUSATORIO) di 2 Sam 11,27b-12,12

\section{Lo svolgimento del rîb (2 Sam 12,13a)}

La parte iniziale-accusatoria presentata sopra ottiene il suo risultato, perché si svolge immediatamente nella confessione di Davide (2 Sam 12,13a) che è la seconda parte della controversia giuridica ${ }^{47}$, o che può essere il secondo atto della liturgia penitenziale ${ }^{48}$ :

"Allora Davide disse a Natan: «Ho peccato contro il Signore!»" (wayyō'mer dāwid 'el-nātāan hạațā'tî̀ layhwh).

La risposta finale di Davide - come si può notare nella Tabella II - per le accuse $\left(A, A^{1}\right.$ e $\left.B, B^{1}\right)$ e per le autoaccuse $\left(C, C^{1}\right)$ è immediata, molto breve ${ }^{49}$ e concreta (essenziale). Il re va d'accordo e si riconcilia con Dio, con il profeta e con se stesso. Nelle sue parole si rivela e si manifesta la sua sincerità, ma anche nello stesso tempo la sua grandezza. La sua colpevolezza dichiarata davanti a se

47 Cfr. ibidem, pp. 23.

48 Cfr. A. Schökel, Trenta Salmi, pp. 211.

49 La risposta di Davide è breve, quasi lapidaria, però molte volte ci sono le risposte dell'accusato assai lunghe, con fenomeni di accumulazioni e di ripetizioni, come ad esempio Sal 51,5-6 (cfr. P. Bovati, Ristabilire la giustizia, pp. 90-91). 
stesso, a Natan e al Signore si rispecchia poi e si sviluppa nel Salmo 51 come suggerisce il suo titolo ${ }^{50}$. Quindi la colpa di Davide si precisa e si svolge nel suo canto rivolto a 'élōhîm, a "Dio" (Sal 51,3a) ${ }^{51}$.

Secondo l'analisi della risposta dell'accusato, fatta da Bovati, nei testi biblici un accusato può confessare la sua colpa a seconda delle tre tipologie distinte che ricevono la seguente formalizzazione: 1 . confessare; 2 . (dire): ho peccato; 3. (dire): tu hai ragione ${ }^{52}$. La confessione di Davide in 2 Sam 12,13a: " "Ho peccato contro il Signore!»” (hâttā'tî̀ layhwh) si classifica nel secondo tipo (gruppo)

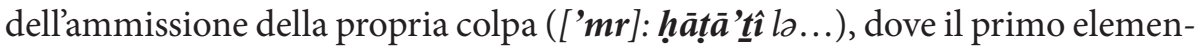
to è un verbo generico che significa "dire" ('mr), mentre il secondo elemento contiene la formula "ho peccato" (hạtâ't tî̀) che tradizionalmente dichiara la propria colpevolezza. È da notare che nel lungo elenco dei testi biblici, presentato dall'autore del "Ristabilire la giustizia", accanto alla confessione davidica di 2 Sam 12,13 si trova anche la dichiarazione individuale del Salmista di

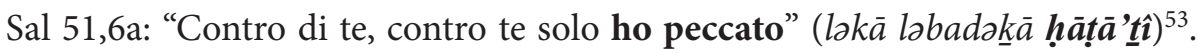
Il verbo caratteristico di questo secondo gruppo è "peccare" (häțā') che viene sostituito qualche volta in alcuni testi attraverso altri verbi oppure tramite altri sostantivi come lo dimostrano per esempio i versetti 3-6 di Sal 51 ${ }^{54}$.

\section{La conclusione della contesa giuridica ( 2 Sam 12,13b-15a)}

I versetti 13b-15a sono la terza e ultima tappa della controversia giuridica in 2 Sam 12, che esprime la riconciliazione tra Dio e Davide. Dio (accusatore) attraverso Natan (accusatore) concede il perdono a Davide (accusato e autoaccusatore). A causa della triplice accusa, Davide si pente, confessa la sua colpa e riceve il perdono dal Signore con la sua promessa di non farlo morire (2 Sam $12,13 b)$ :

"Il Signore ha perdonato il tuo peccato; tu non morirai"

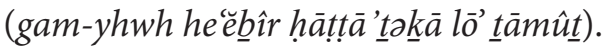

Il processo è finito. Laccusato si è riconciliato con l'accusatore. Ha ricevuto il perdono (vedi Tabella I). Rimane solo l'espiazione, la riparazione del torto.

\footnotetext{
50 Cfr. G. Ravasi, Il Libro dei Salmi (II), pp. 19.

51 Cfr. ibidem, pp. 19-20.

52 Cfr. P. Bovati, Ristabilire la giustizia, pp. 78-103.

53 Cfr. ibidem, pp. 86-88.

54 Cfr. P. Ravasi, Il Libro dei Salmi (II), pp. 19.
} 
Il colpevole deve pagare la sua ingiustizia: "il suo figlio [...] dovrà morire" (2 Sam 12,14b) e morirà (2 Sam 12,18). In queste parole si realizza la fine del processo forense che si compone della sentenza (2 Sam 12,13b-14) e della sua esecuzione (2 Sam 12,18$)^{55}$.

Prendendo in considerazione lo studio scientifico sulla sentenza nella letteratura biblica proposta da Bovati ${ }^{56}$ e applicandolo nell'analisi di 2 Sam 11,27b$-12,15$ a si possono notare in questa controversia giuridica, oltre le due sentenze presentate sopra (13b e 14b di 2 Sam 12), anche le altre quattro (5b, 6a, 7a e 10a di 2 Sam 12).

Nella divisione fatta dall'autore del "Ristabilire la giustizia", il primo tipo di sentenza ha la forma "tu sei colpevole/innocente" pronunciata dal giudice e rivolta a un accusato (qualcuno) ${ }^{57}$. Sembra che a questo gruppo di sentenza appartengano le parole pronunciate da Natan a Davide, già analizzate prima come accusa (2 Sam 12,7a): “Tu sei quell'uomo!" ('attāâh hā'î̌s $)^{58}$.

Il secondo tipo di sentenza si definisce nel modo seguente: "è reo di ... (morte)", pronunciata dal giudice riguardo a un accusato (qualcuno ${ }^{59}$. In questa seconda modalità si presenta la risposta di Davide dopo aver udito la parabola (fittizia) di Natan (2 Sam 12,5b): "Perché quell'uomo ha fatto questo merita la morte" (kî ben ${ }^{60}-m \bar{a} w e \underline{t} h \bar{a}^{\prime} \hat{i s ̌}$ hāośśeh zōt).

Nella terza ed ultima modalità di sentenza, più usata, il giudice condanna (o assolve, perdona) un accusato (qualcuno) a morte. Spesso la formula utiliz-

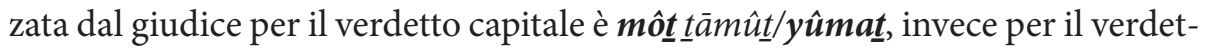
to di proscioglimento è $\mathbf{l} \overline{\boldsymbol{o}} \underline{\underline{t}} \overline{\boldsymbol{a}} \boldsymbol{m} \mathbf{u} \underline{t}^{61}$. Entrambe le sentenze sono tra loro vicine nella sezione profetica di 2 Sam quando Dio risparmia Davide (v. 13b): "tu non

55 Cfr. P. Bovati, Ristabilire la giustizia, pp. 317; G. Ravasi, Samuele, pp. 1418 ("Dio perdonando la colpa, deve essere il garante della giustizia e quindi deve dar corso alla pena: Davide sarà punito come padre perdendo il figlio avuto da Betsabea. La morte del figlio - nella visione veterotestamentaria - diventa quasi il simbolo della morte del re peccatore proprio nella sua stessa carne. Ma la nascita di Salomone sarà segno della «rinascita» di Davide e del suo ritorno alla comunione con Dio e con la sua giustizia").

56 Cfr. P. Bovati, Ristabilire la giustizia, pp. 317-357.

57 Cfr. ibidem, pp. 328-329.

58 Vedi la nota 25 di questo articolo.

59 Cfr. P. Bovati, Ristabilire la giustizia, pp. 330 (Questo secondo tipo di sentenza ha la sua triplice divisione con la propria fraseologia ebraica: a) ben-māwet; b) mišpāt māwet; c) dām/dāmîm spesso con $r o ̄ s s)$.

60 Cfr. ibidem, pp. 330 ("La prima forma [di questo secondo gruppo di sentenza] ha nel sostantivo ben il termine che definisce la condizione del reo, termine che grammaticalmente regge un altro sostantivo indicante la sanzione").

61 Cfr. ibidem, pp. 332-334. 


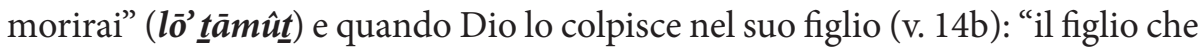
ti è nato dovrà morire" (gam habbēn hayyillôd laḳā môt ya ämût). La sentenza di questo terzo tipo, per ragioni letterarie o altre può essere sostituita da unaltra formula come si vede ad esempio in 2 Sam 12,10a: "Ebbene, la spada non si

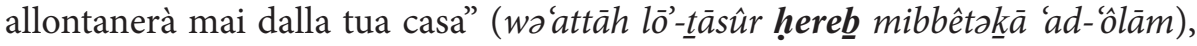
dove la spada è un'allusione alla morte sanguinosa di Amnòn, Assalone e Adonia, i tre figli di Davide ${ }^{62}$. Laltra sostituzione di questo terzo gruppo di sentenza è resa con la formula che concerne il risarcimento (dei danni) con il verbo tipico šlm $(\mathrm{Pi})$ che si trova in 2 Sam 12,6a: "pagherà quattro volte" (yəšallèm 'araba'sttāyim) e che viene trattata anche nel paragrafo 2.3 come autoaccusa ${ }^{63}$.

\section{Conclusione}

Lo studio di 2 Sam 11,27b-12,15a, fatto in questo articolo, presenta un bellesempio del rîb che è una controversia, la quale viene creata fra le due parti giuridiche e costituita dalle tre fasi (o tre elementi): accusa, risposta dell'accusato e riconciliazione (cf. Tabella I). Nella fase iniziale-accusatoria della controversia si trova la triplice imputazione della contesa con la sua gradazione: Dio, Natan, Davide. La parte accusatoria dei versetti 11,27b-12,12 di 2 Sam è molto ricca e interessante dal punto di vista del rîb e del giudizio (cf. Tabella II). "Ciò che Davide aveva fatto (l'adulterio con Betsabea e l'uccisione di suo marito) era male agli occhi del Signore" (2 Sam 11,27b; 12,9b). Per questo Dio manda il profeta Natan, come suo messaggero, a Davide per accusarlo (2 Sam 12,1a). Natan, nella sua parabola fittizia presenta, da una parte, la causa (come avvocato) al re, supremo giudice, e dall'altra, lo accusa, mostrandogli, in modo nascosto (sfumato), il suo reato (delitto) perverso (2 Sam 12,1b-4). Davide risponde con la doppia sentenza (2 Sam 12,5b.6a) che diventa autogiudizio o meglio autoimputazione, se si accetta tutto il contesto accusatorio (2 Sam 11,27b-12,12) e la sentenza finale di Dio (2 Sam 12,13b-14). Lapplicazione improvvisa di Natan: "Sei tu quell'uomo!" (12,7a) contemporaneamente scopre la colpevolezza di Davide e lo accusa. Ma tutto ciò diventa, per Davide, la sua svolta. Il triplice processo accusatorio in $2 \mathrm{Sam}(\mathrm{A}=11,27 \mathrm{~b} ; \mathrm{B}=12,1 \mathrm{~b}-4 ; \mathrm{C}=12,5 \mathrm{~b})$ con la sua gradualità (Dio, profeta, re) e con la sua ripetizione $\left(\mathrm{A}^{1}=12,7 \mathrm{~b}-12 ; \mathrm{B}^{1}=12,7 \mathrm{a}\right.$; $\left.\mathrm{C}^{1}=12,6 \mathrm{a}\right)$ conduce subito alla confessione di Davide $(12,13 \mathrm{a})$, cioè alla risposta dell'accusato, che è la seconda parte della controversia giuridica. Mentre i ver-

62 Cfr. ibidem, pp. 332-333; La Bibbia di Gerusalemme, Bologna 222008, pp. 581.

63 Cfr. P. Bovati, Ristabilire la giustizia, pp. 333-334. Vedi anche le note 27 e 28 di questo articolo. 
setti 13b-14 sono la terza e ultima tappa del rîb in 2 Sam 12 che esprimono la riconciliazione tra Dio e Davide. Dio (accusatore) attraverso Natan (accusatore) concede il perdono a Davide (accusato e autoaccusatore).

Dall'analisi di 2 Sam 11,27b-12,15a risulta che Dio, nel rîb, non si rivela come giudice, fra le due parti in conflitto, ma come accusatore, che non vuole vincere umiliando l'accusato, ma convincerlo e correggerlo. Egli si comporta come membro, legato di una alleanza, che desidera piuttosto salvare che condannare il suo alleato colpevole. Davanti all'accusatore misericordioso l'accusato ingiusto si apre e si dichiara reo, ammette la propria colpa e riceve alla fine il perdono. Il processo del rîb si conclude con la riconciliazione tra le due parti.

Riconoscere e confessare il proprio peccato, come ha fatto Davide (2 Sam 12,13a), è un atto di glorificazione del Signore, perché la confessione dei peccati diventa anche la confessione di fede in Dio. Chi si confessa compie il più bel sacrificio di lode per Dio e nello stesso tempo manifesta il migliore atteggiamento davanti al Signore, perché la grandezza dell'uomo si esprime proprio nel dire la verità su se stesso. Il cuore contrito, aperto, sincero, umile durante la confessione non solo invoca il perdono di Dio, ma anche permette a Dio di salvare e liberare il peccatore dalle conseguenze del peccato e dalle sue sventure. Ecco il vero volto della misericordia divina che si basa sulla giustizia umana in conformità con la giustizia divina e scaturisce dalla controversia giuridica, dal rîb.

\section{Bibliografia}

Anderson A.A., 2 Samuel (WBC 11), Dallas 1989.

Auld A.G., I \& II Samuel, Louisville 2011.

Auld A.G. - Eynikel E., For and against David: Story and History in the Books of Samuel, Leuven 2010.

Bodi D., The demise of the warlord: a new look at the David story, Sheffield 2010. Bovati P., I rîb profetici, Roma 1997. , Ristabilire la giustizia. Procedure, vocabolario, orientamento, Roma 2005.

Costacurta B., Lo scettro e la spada. Davide diventa re (2 Sam 2-12), Bologna 2006.

Firth D., 1 and 2 Samuel: A Kingdom Comes, Sheffield 2013.

Gioda S., Dizionario, sinonimi e contrari, Roma 1992.

Gordon R.P., 1 \& 2 Samuel, Sheffield 1984.

Haag H., Dizionario biblico, Torino 1960.

Janzen D., The condemnation of David's "taking" in 2 Samuel 12:1-14, JBL (2012), pp. 209-220.

Jones G.H., The Nathan narratives (JSOT Sup 80), Sheffield 1990.

Joüon P.P., Grammaire de l'hébreu biblique, Roma 1987 (ristampa).

Koenig S.M., Isn't this Bathsheba? A study in characterization, Eugene 2011. 
Lambdin T.O., Introduction to biblical Hebrew, London 1990.

McCarter P.K., II Samuel (AB 9), New York 1984.

McKenzie J.L., Dizionario biblico, Assisi 1978.

Oswald W., Nathan der Prophet: Eine Untersuchung zu 2 Samuel 7 und 12 und 1 Könige 1 , Zürich 20.

Ravasi G., Il Libro dei Salmi, vol. I, Bologna 1981. , Il Libro dei Salmi, vol. II, Bologna 1988. , Samuele, in: Nuovo Dizionario di Teologia Biblica, Torino 1988, 1418.

Schipper J., Did David overinterpret Nathan's parable in 2 Samuel 12:1-6?, JBL 126.2 (2007), pp. 383-407.

Schökel A., Trenta Salmi, Bologna 1982.

Sicre J.L., I profeti d'Israele e il loro messaggio, Roma 1989.

Simon H., The poor man's ewe-lamb. An example of a juridical parable, Bib 48 (1967), pp. 207-242.

Vella J., La giustizia forense di Dio, Brescia 1964.

Vogels W., David et son histoire. 1 Samuel 16,1 - 1 Rois 2,11, Montréal 2003. 